\title{
Effect of UV-C Hormesis in Regulating Anthracnose Disease and Postharvest Quality of Tomato
}

\author{
W. U. T. Wanasinghe and J. W. Damunupola* \\ Date Received: $11^{\text {th }}$ November 2019 / Date Accepted: $24^{\text {th }}$ February 2020
}

\begin{abstract}
Purpose : Anthracnose disease caused by Colletotrichum coccodes is a significant postharvest disease in tomatoes. Although there are negative impacts of fungicides usage on fresh produce, at present, it is the most common method of eliminating diseases. UV-C hormesis was used in this study as a trending non-chemical postharvest treatment.

Research Method: Tomato varieties 'Thilina' and 'Roma' were used to check the efficacy of UV-C treatments. Five different $U V$-C dosages $\left(0,1,2,3,4,5 \mathrm{~kJ} / \mathrm{m}^{2}\right)$ were applied to pure cultures of $C$. coccodes (254 $\mathrm{nm}$ at a distance of 15 $\mathrm{cm})$. The selected dosages ( 3.0 and $4.0 \mathrm{~kJ} / \mathrm{m}^{2}$ ) were tested on the effect of anthracnose disease development in vivo and the change in postharvest quality parameters (weight loss, firmness, shelf life, antioxidant and total phenolic content). Antifungal activity of the peel was investigated using Cladosporium bioassay.

Findings : Weight loss of treated 'Thilina' variety has significantly increased $(P<0.05)$. The antioxidant activity and the total phenolic content of both treated varieties have increased. The firmness has significantly retained in treated 'Thilina' variety. Further, the shelf life has significantly extended in both treated varieties by approx. 2 folds compared to the non-treated control. All tested tomato samples indicated the presence of antifungal compounds in their peels. Therefore, to suppress anthracnose disease $4.0 \mathrm{~kJ} / \mathrm{m}^{2}$, and 3.0 and 4.0 $\mathrm{kJ} / \mathrm{m}^{2}$ are the most suitable UV-C dosage for 'Thilina' and 'Roma' respectively.
\end{abstract}

Research Limitations : This research is based on the available tomato cultivars in the market.

Originality/Value : This is a non-chemical treatment which could be utilized by farmers in future, to control postharvest anthracnose in tomatoes.

Keywords: Anthracnose, Colletotrichum, Postharvest, Tomato, UV-C hormesis

\section{INTRODUCTION}

Tomato fruit (Solanum lycopersicum), commonly considered as a vegetable, is a very popular and highly consumed fresh produce globally. Tomatoes provide a great deal of nutrients and other health-related food components, for humans. They are a rich source of folate, vitamin $\mathrm{A}, \mathrm{C}$ and $\mathrm{E}$, flavonoids, phytosterols and potassium (Agarwal, 2000; Beecher, 1998). The antioxidant capacity of tomato is mostly attributed to carotenoids and out of many carotenoids, lycopene is the most prominent carotenoid present in tomato.

Postharvest fresh produce face many detrimental changes during storage including diseases caused by pathogens such as fungi and bacteria, reduction in antioxidant activity and early senescence of fruits, which ultimately lead to reduced shelf life. Tomato fruit is also susceptible to many diseases caused by fungi. Among the diseases, anthracnose disease caused by C. coccodes is one of the major tomato postharvest diseases in Sri Lanka. Fungal diseases can result in serious economic losses to fruit and vegetables, both from the pre- and postharvest damages (Wills et al., 2007). Such

\footnotetext{
* Department of Botany, Faculty of Science, University of Peradeniya, Sri Lanka.

jilushi@yahoo.com
}

(1) ORCID http://orcid.org/0000-0001-7427-5582 
diseases are commonly controlled by the use of commercial fungicides. However, such chemical treatments are restricted in most countries, due to increasing concerns about chemical residues on the edible fruit. Many countries have imposed quality regulations to ensure high quality produce with minimum chemical residues in fresh produce. Therefore, safe alternative control methods need to be developed.

Different techniques have been investigated to extend postharvest life of fresh produce such as the use of refrigerated storage, controlled and modified atmosphere, heat treatments, application of 1-MCP, etc. (Wills et al., 2007). During past few decades much attention has focused on induction of natural disease resistance (NDR) in harvested horticultural crops using physical, biological and/or chemical elicitors (Terry and Joyce, 2004). It is stated that beneficial plant responses can be induced by low or sub-lethal doses of an elicitor/agent. Recently, the use of such elicitors/agents in improving favorable plant responses leading to plant protection has been widely recognized (Kuc', 2000).

This study aims to select a suitable UV-C treatment to suppress the anthracnose disease in most commonly used local tomato varieties in Sri Lanka and to investigate the effect of UV-C treatment on physical and chemical parameters on postharvest quality of tomato fruits. Although there are many studies correlating UV-C treatments on tomato (Onik, et al., 2019; Pinheiro et al., 2015), regulating nutrient parameters and antioxidant activity in fruits, this is the first reported study with UV-C treatments tested on commonly consumed Sri Lankan tomato varieties to investigate the effect on in vivo and in vitro anthracnose pathogen behaviour along with the regulation of postharvest quality parameters.

\section{MATERIALS AND METHODS}

\section{Determination of the natural disease resistance of selected tomato varieties in Sri Lanka}

The tomato varieties: 'Lanka Sour', 'Rashmi' and 'Bhathiya F1 hybrid' were obtained from
Horticultural Crop Research and Development Institute (HORDI), Gannoruwa, Sri Lanka. Variety 'Thilina' was purchased from local central market while 'Roma' variety was purchased from a super market, Kandy, Sri Lanka. Eight to five tomatoes (of uniform size and without any defects) per variety, at mature green stage were selected and surface sterilized with $70 \%$ ethanol. These fruits were initially kept in moistened chambers for 24 hrs and then they were randomly transferred to ripening boxes for natural diseases development and the disease percentage was determined at three days intervals. Experiment was initiated within a day or two after the harvesting process. The best variety for UV-C treatment was selected for subsequent experiments (variety/varieties with less natural disease resistance).

\section{Analysing the presence of fungicidal residues on tomato}

The Cladosporium bioassay was performed according to the procedure described by Kanatiwela \& Adikaram (2009) with slight modifications. A $30 \mathrm{ml}$ (deionized water) aliquot of washing per 3 tomato fruits were obtained using a magnetic stirrer after agitating for 20 minutes. Aliquots $(50 \mu \mathrm{L})$ were spotted on freshly activated TLC plates and air-dried at room temperature $\left(25^{\circ} \mathrm{C}\right)$ and subjected to Cladosporium bioassay. Dithane M-45 (mancozeb) was used as the positive control and deionized water was used as the negative control. The plate was immediately placed in a moisture chamber $\left(100 \% \mathrm{RH}\right.$ and $\left.25{ }^{\circ} \mathrm{C}\right)$ for incubation for 2-3 days until green mycelial growth was visible. Areas showing absence of mycelial growth were taken as inhibition zones (Kanatiwela \& Adikaram, 2009) and the diameters of such zones were measured.

\section{Determination of the effect of $U V$-C treatment on $C$. coccodes (in vitro)}

Pure cultures (obtained from Institute of Postharvest Technology, Anuradhapura) of C. coccodes were cultured on potato dextrose agar 
medium (PDA). A 6 mm mycelial plug was taken from pure cultures and kept on freshly prepared PDA plates and they were allowed to stabilize for $48 \mathrm{hrs}$ before giving the treatment. Five different UV-C dosage treatments $(0,1,2,3,4$ and $5 \mathrm{~kJ} /$ $\mathrm{m}^{2}$ ) were applied to prepared pure cultures at an intensity of $254 \mathrm{~nm}$ (at a distance of $15 \mathrm{~cm}$ ). The different dosages were calculated according to the equation in Darras et al., 2010. The UV-C lamp (model: BBS-V1300) had a nominal power output of $5256.67 \mu \mathrm{W} \mathrm{cm}$ ch $^{-2}$ and peak wavelength emission of $254 \mathrm{~nm}$. Three replicates per treatment were used. Mycelial growth area of the treated culture plates were recorded at two day intervals. Effects of the UV-C treatment on colony growth of the treated plates were compared with the $0 \mathrm{~kJ} / \mathrm{m}^{2}$ dosage (non-treated control). Finally, the treatment with the greatest suppression of the colony growth was selected as the best treatment for in vivo studies.

\section{Investigation of the efficacy of selected $U V-C$ treatment on tomatoes (in vivo)}

Disease free 'Thilina' and 'Roma' tomato varieties were used (36 fruits per trial from each variety). Three fruits per replicate were randomly selected for each treatment. Surface sterilized fruits with $70 \%$ ethanol were inoculated with mycelium plugs of 7-10 days old pure cultures of $C$. coccodes. These plugs were placed/ wound inoculated on the fruit surface at two previously marked spots per fruit. For wound inoculation, a $\sim 2 \mathrm{~mm}$ depth wound was made using a sterile tip of a scalpel blade. Fruits were irradiated under UV-C light. Treatments were done before the inoculation for 18 fruits and after the inoculation for the other 18 fruits. For both inoculations, the effect of wounding and non-wounding was tested as per given in Figure 01. The treatments were given after 2 days of inoculation for, before treatment inoculation process. Effective UV-C dosages were selected based on the in vitro experimental results $\left(3.0\right.$ and $\left.4.0 \mathrm{~kJ} / \mathrm{m}^{2}\right)$.

\section{Analyzing the postharvest quality of $U V-C$ treated tomatoes}

To analyze the postharvest quality, both 'Roma' and 'Thilina' tomatoes treated with $3.0 \mathrm{~kJ} / \mathrm{m}^{2}$ and $4.0 \mathrm{~kJ} / \mathrm{m}^{2}$ were taken.

Physical properties: As the physical properties, weight loss and firmness was measured. Weight loss of the tomatoes was determined by weighing the individual fruits at the beginning and during storage. Results were expressed as a percentage of weight loss relative to the initial fresh weight.

Percentage weight loss $=$

$\frac{\{\text { Initial fresh weight }(\mathrm{g}) \text {-Final fresh weight }(\mathrm{g})\}}{\text { Initial fresh weight }(\mathrm{g})} * 100$

The firmness was determined using a fruit penetrometer.

Chemical properties: Antioxidant activity was determined using DPPH assay (Liu et. al., 1993). The absorbance was measured at $517 \mathrm{~nm}$ using a UV-VIS Spectrophotometer. The total phenol content was determined by the Folin-Ciocalteu method using Gallic Acid (GA) as the standard. The standard curve was prepared using working solution of Gallic Acid.

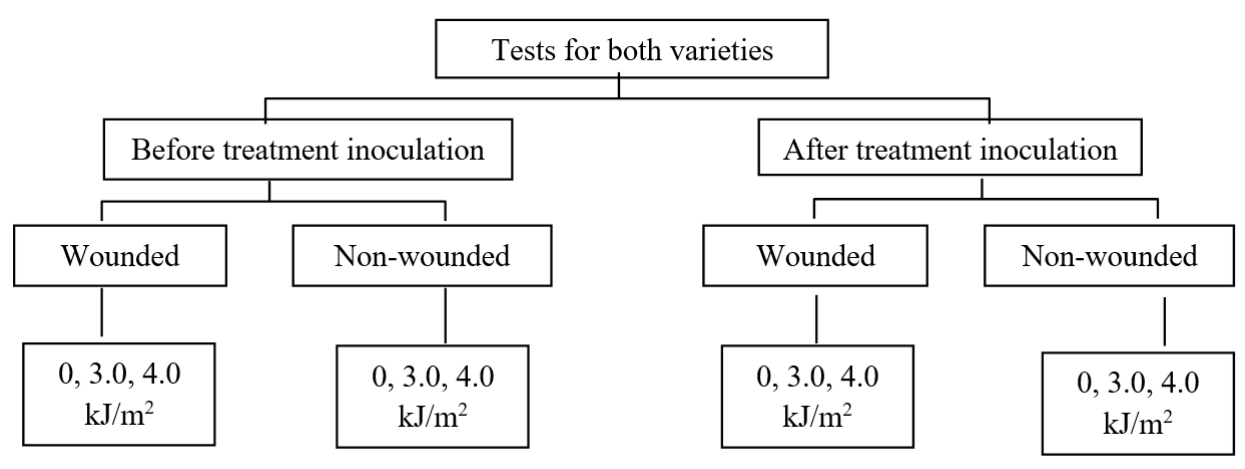

Figure 01: Flow chart to show different treatments tested to investigate the efficacy of selected UV-C treatment on tomatoes. 
Postharvest longevity: The shelf life was determined by considering the deterioration of the fruit. Deterioration was determined by observing, discoloration, fruit rotting and shrivelling of the peel.

\section{Statistical analysis}

All data were analyzed using one-way ANOVA, where Fisher's least significant difference procedure was used to compare treatments $(\mathrm{P}=0.05)$ using Minitab ver.17.1.0. Graphs were constructed using SigmaPlot ver.10.0. All the experimental trials were repeated at least once.

\section{RESULTS AND DISCUSSION}

Tomatoes face many detrimental changes during postharvest storage. These include induction of diseases caused by pathogens such as fungi and bacteria, reduction in antioxidant activity and early senescence of fruits, which ultimately lead to decreased postharvest longevity. However, if a fruit stays longer time period without/ less diseases, that fruit could be considered as a one with more natural disease resistance. In this study, after 12 days of observations, it was revealed that 'Bhathiya' and 'Rashmi' varieties have more natural disease resistance than the other varieties of tomato (Figure 2). 'Lanka Sour' has the least natural disease resistance (highest disease percentage of $100 \%$ ) among the varieties selected (Figure 02). Since 'Lanka Sour' has very low consumer preference, 'Thilina' and
'Roma' varieties, which showed high disease susceptibility and also commonly consumed by local people, were used throughout the study.

TLC Cladosporium bioassay easily detects the presence of antifungal compounds. The procedure is simple, yet sensitive, precise, versatile, rapid and inexpensive (Kanatiwela \& Adikaram, 2009). In this study, all the washing samples used for the test gave positive results for the presence of antifungal compounds. The inhibition zones indicate the presence of antifungal compounds on the tomato washings, because the presence of substances toxic to Cladosporium is indicated by the lack of aerial mycelium. This implies that there are antifungal residues present in the tomato fruit washings tested. Therefore, it is vital to seek possible ways to reduce the usage of synthetic fungicides on fresh produce.

The results shown in Figure 03 indicate that, postharvest UV-C treatments had significant differences on the colony growth of $C$. coccodes compared with the control $\left(0 \mathrm{~kJ} / \mathrm{m}^{2}\right)$. Control treatment $\left(0 \mathrm{~kJ} / \mathrm{m}^{2}\right)$ showed the highest mean colony growth $(5.93 \pm 0.73)$ compared to other dosages (Figure 04). Significantly, the least mean value $(2.533 \pm 0.13)$ for the colony growth was shown at the dosage of $4.0 \mathrm{~kJ} / \mathrm{m}^{2}$. The dosage, which showed the least colony growth, was selected as the best dosage to treat tomato fruits (in vivo experiment). Thus, at $25{ }^{\circ} \mathrm{C}$, the most effective UV-C dosage against the growth of $C$. coccodes was $4.0 \mathrm{~kJ} / \mathrm{m}^{2}$. Shama and Alderson (2005) emphasized that, low doses of the harmful agents can induce stress conditions in living organisms.

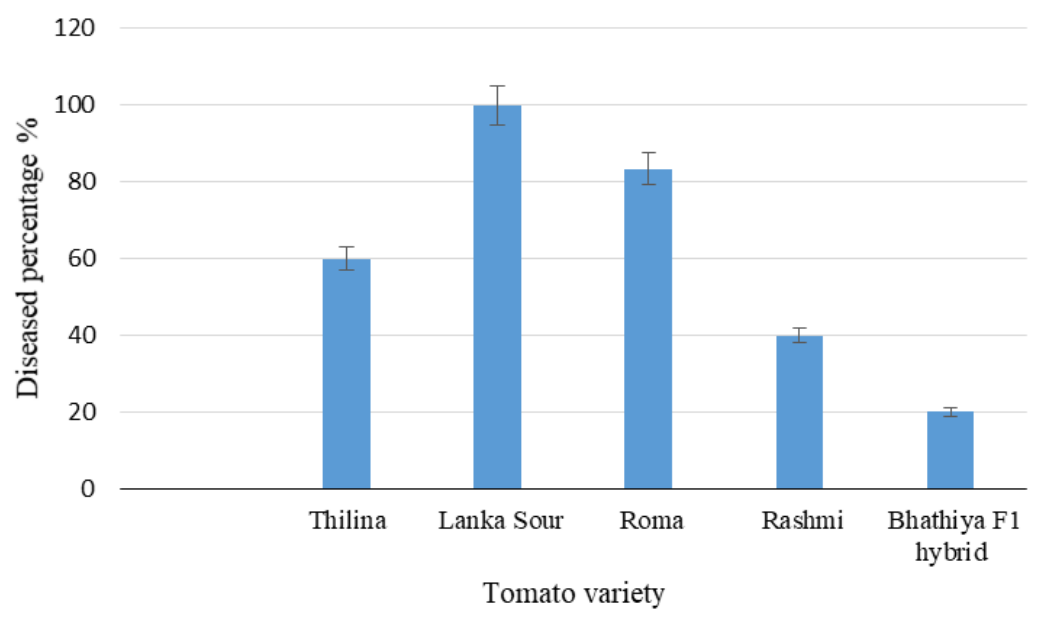

Figure 02: Diseased percentages of different tomato varieties (Average $\pm \mathrm{SE}$ ) after 12 days. 


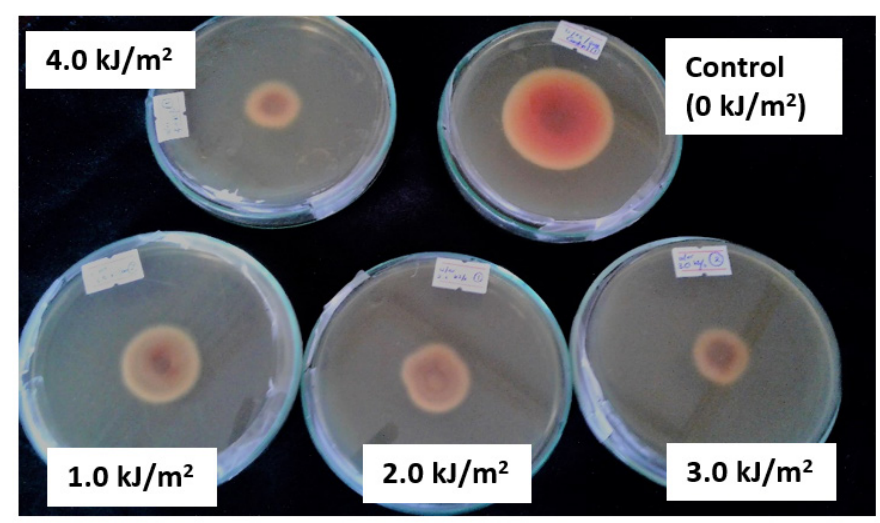

Figure 03: The effect of selected UV-C treatments on $C$. coccodes colony growth.

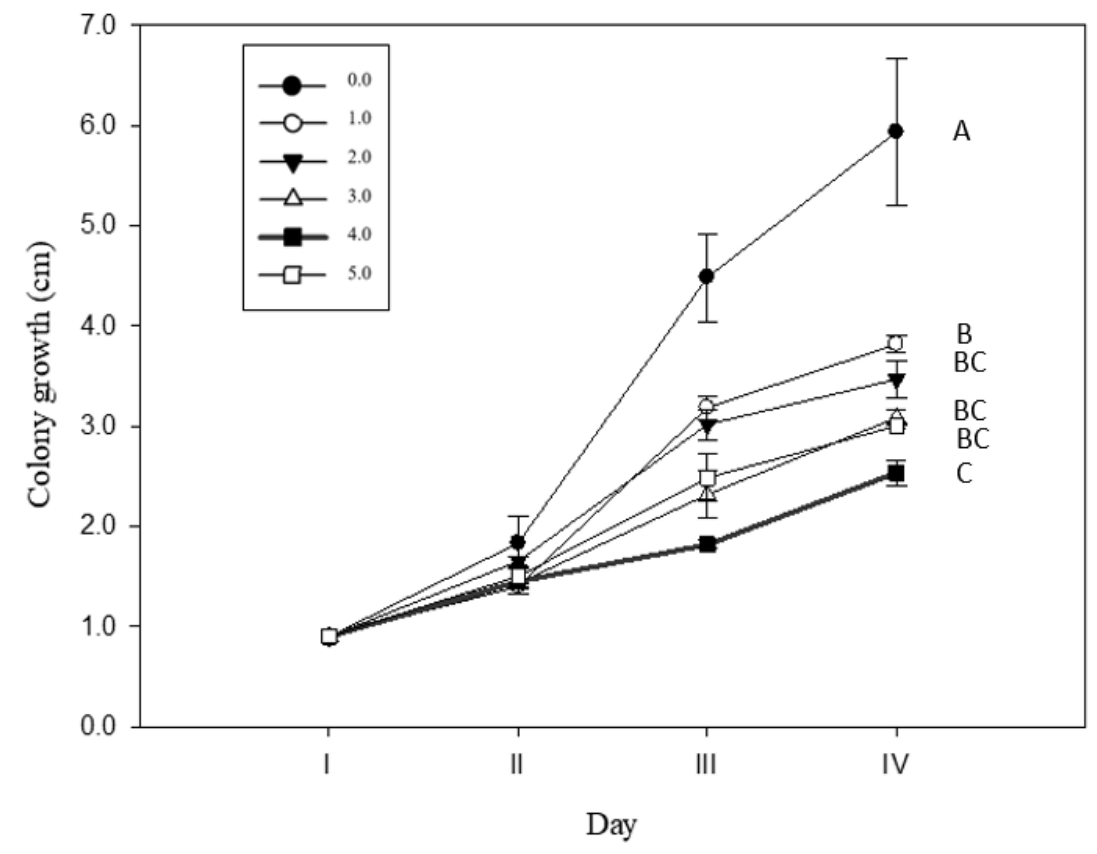

Figure 04: Effect of different UV-C dosage treatments on the mean colony growth of $C$. coccodes in vitro $\left(0,1.0,2.0,3.0,4.0\right.$ and $\left.5.0 \mathrm{~kJ} / \mathrm{m}^{2}\right)$. Different letters indicate the significance of the treatments.

UV light alters the growth of the fungus by changing DNA structure of the cells and damaging walls of the cells. High dosages of irradiation directly damage the DNA of living organisms inducing cross-linkages, which make the organism unable to grow or reproduce. Further it also affects the spore production and spore germination (Tauxe, 2001). The lowest but most effective dosage was selected as the best UV-C dose to treat the tomatoes. Therefore, the most effective dose and the one prior to that (4.0 and $3.0 \mathrm{~kJ} / \mathrm{m}^{2}$, respectively) were selected to treat the fresh fruits.

A direct indication of the growth of the fungus after inoculation of fruit is given by the measurement of the diameter of fungal lesion. During the in vivo experiment, three main parameters of applying UV-C treatment for two varieties were compared: effect of the treatment time (before inoculating treatments and after inoculating treatments), effect of the wounding and non-wounding and the effect of dosage $(0$, 3.0 and $4.0 \mathrm{~kJ} / \mathrm{m}^{2}$ ) (Figure 05).

According to the statistical analysis, in both varieties, there is no significant difference in the effect of treatment time $(\mathrm{P}>0.05)$. However, there is a significant difference in the effect of wounding in 'Thilina' $(\mathrm{P}<0.05)$. In variety 'Roma', the wounding effect did not vary significantly $(\mathrm{P}>0.05)$. That might be 
due to the fact that 'Roma' variety possesses a comparatively hard peel than the 'Thilina' variety. 'Thilina' showed a significant difference for $4.0 \mathrm{~kJ} / \mathrm{m}^{2}(\mathrm{P}<0.05)$ when compared to the control. But there is no significant difference between $3.0 \mathrm{~kJ} / \mathrm{m}^{2}$ treatment $(\mathrm{P}>0.05)$ and the control. The suppression occurs through two main means: direct inactivation and induced effect. Direct fungal inactivation occurred once a sufficiently high UV dose had been accumulated by the organism. That caused changes in DNA of the fungus (Tauxe, 2001). The treatment of fruit with low UV doses results in the synthesis of a number of antifungal compounds in the fruit. It could enhance the phytoalexin production, the induction of phenylalanine amonia lyase (PAL) and the induction of pathogenesis-related (PR) proteins (chitinases and $\beta-1,3$-endoglucanases) (Shama and Alderson, 2005). This supports that
UV can be responsible in mediating these gradual biochemical defence responses (Mditshwa et al., 2017) in the peel of UV irradiated fruit.

In variety 'Roma', the percentage weight loss is significantly $(\mathrm{P}<0.05)$ less when treated with UV-C. However, in 'Thilina', there is no significant difference in percentage weight loss after treatments $(\mathrm{P}>0.05)$ (Figure 06). Tomatoes are usually sold on weight basis and therefore, increased weight loss negatively affects the market prices. Weight loss naturally occurs during ripening. The accumulation of reactive oxygen species (ROS), microbial growth, cell wall degrading enzymes and higher respiration rate are the prominent changes leading to a quality loss in tomato fruit. These changes in turn lead to texture loss, weight loss, physiological disorders and decay (Mditshwa et al., 2017; Wills et al., 2007).

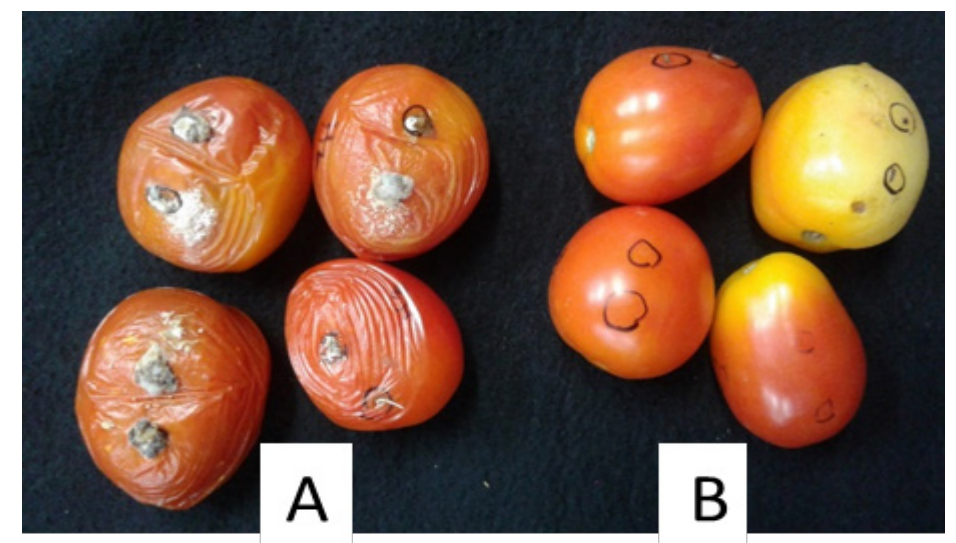

Figure 05: Effect of the UV-C treatment on 'Roma' (A) Non-wounded, $0 \mathrm{~kJ} / \mathrm{m}^{2}$ and (B) Non-wounded, $4.0 \mathrm{~kJ} / \mathrm{m}^{2}$ after 6 days of treatment.

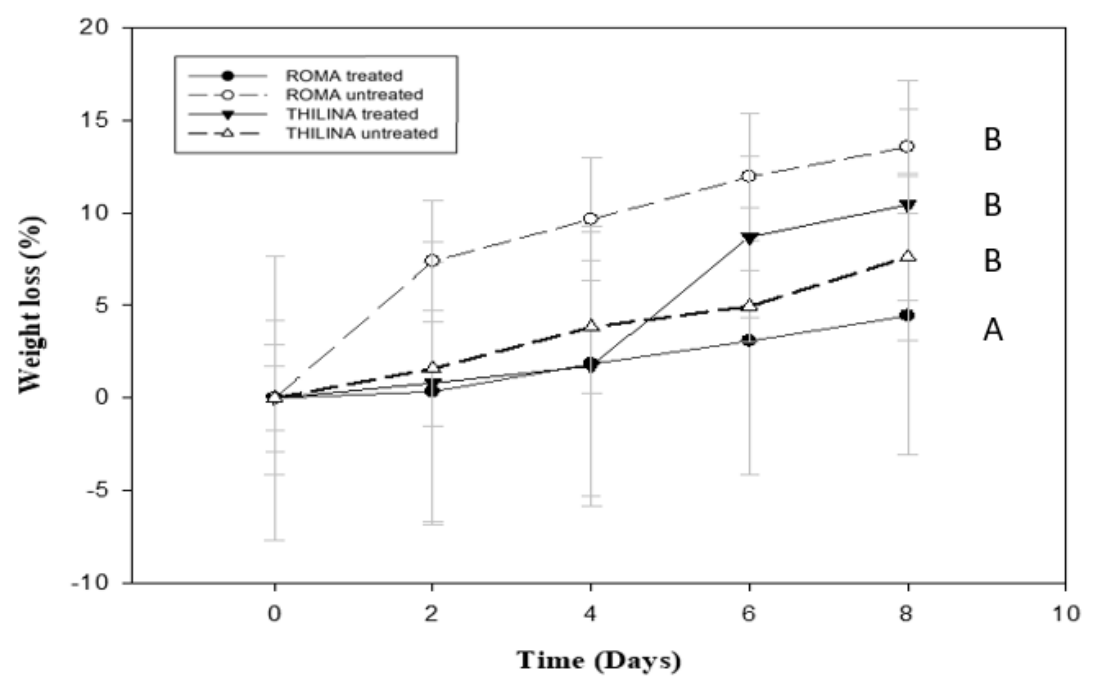

Figure 06: Effect of UV-C treatment on weight loss. Different letters indicate the significance $(\mathrm{P}<$ $0.05)$ of the treatments. 


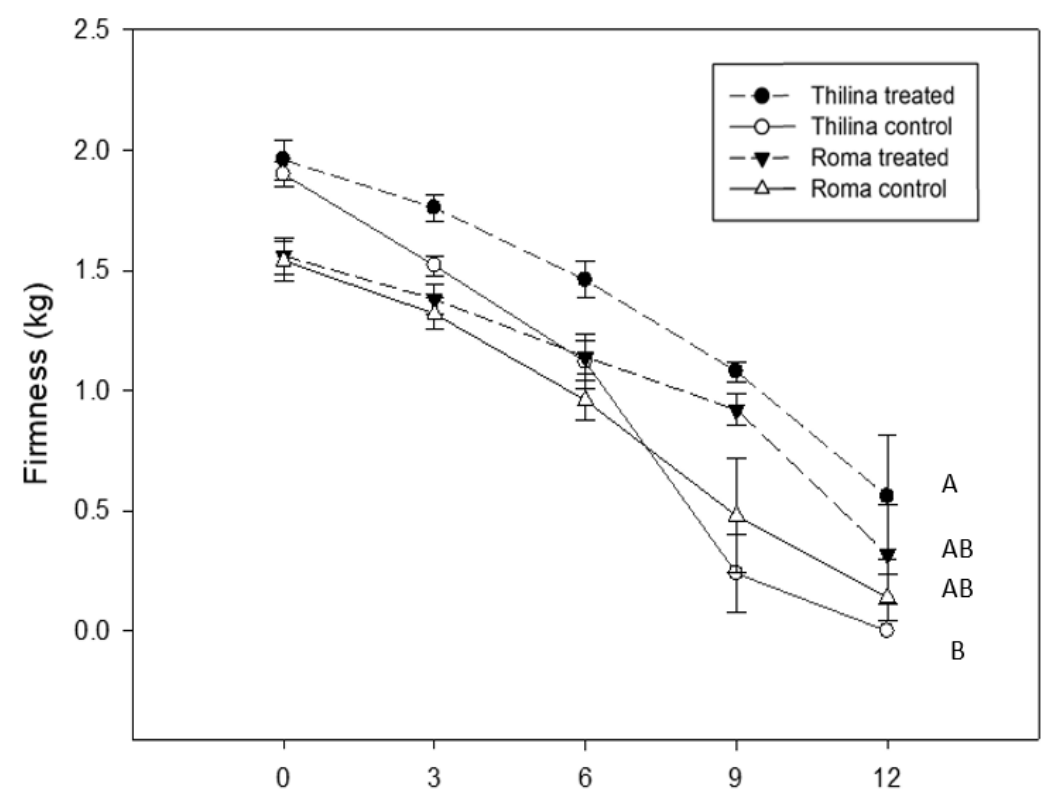

Figure 07: Effect of UV-C treatment on firmness on tested tomato cultivars. Different letters indicate the significance $(\mathrm{P}<0.05)$ of the treatments.

According to the results, UV-C treatment has shown higher firmness retention compared to the untreated fruits. As per the Figure 07 given below, firmness of 'Thilina' variety, has retained significantly $(\mathrm{P}<0.05)$ when UV-C treated. However, in 'Roma', there is no significant difference in firmness after treatment $(\mathrm{P}>0.05)$ (Figure 07). Fruit firmness decreases due to fruit softening while ripening. Fruit softening could arise from one of the three mechanisms: loss of turgor; degradation of starch; or breakdown of the fruit cell walls due to fungal attack. UV-C reduces cell wall degradation of the pericarp (Mditshwa et al., 2017). Previous research has shown that UV-C significantly inhibits cellulase, polygalacturonase and pectin-methyl-esterase activities, the key cell wall degrading enzymes, resulting in higher firmness retention ( $\mathrm{Bu}$ et al., 2013).

Both 'Thilina' and 'Roma' varieties have shown an increase in antioxidant activity with the $\mathrm{UV}-\mathrm{C}$ treatment. The $\mathrm{IC}_{50}$ values of both treated varieties have decreased, than those of the nontreated. The lower the $\mathrm{IC}_{50}$ value, the higher the antioxidant activity. The ascorbic acid content of tested tomatoes had increased with the increase in storage period and ripening time (Cantwell, 2000). The change may be due to that, UV-C light stresses the plant tissue and that stimulates the biosynthesis of secondary metabolites, which includes compounds with antioxidant activity (Liu et al., 2012).

Gallic acid was the most abundant phenolic compound in tomato fruit, and usually it increases during storage (Mditshwa et al., 2017). In our study, treated fruits of both varieties, have higher total phenolic content compared to non-treated fruits (data not shown). This may be due to that, UV light triggers the accumulation of UVlight absorbing flavonoids and other phenolic compounds (Liu et al., 2012). After UV-C treatments, enzymes like phenylalanine ammonia lyase (PAL) can get activated. It acts as a catalyst for the synthesis of phenolic compounds such as phenylpropanoids, coumarins and flavonoids (Mditshwa et al., 2017).

According to the results shown in the graph (Figure 08), a significant extension of shelf life was observed in both UV-C treated 'Thilina' and 'Roma' varieties $(\mathrm{P}<0.05)$. The longer shelf life was observed in treated 'Thilina' variety, than in 'Roma'. In variety 'Thilina', the treatments enhanced the postharvest longevity approximately by two fold, compared to the nontreated control after two weeks ( $\sim 7$ days in nontreated versus 13.5 days in treated). 


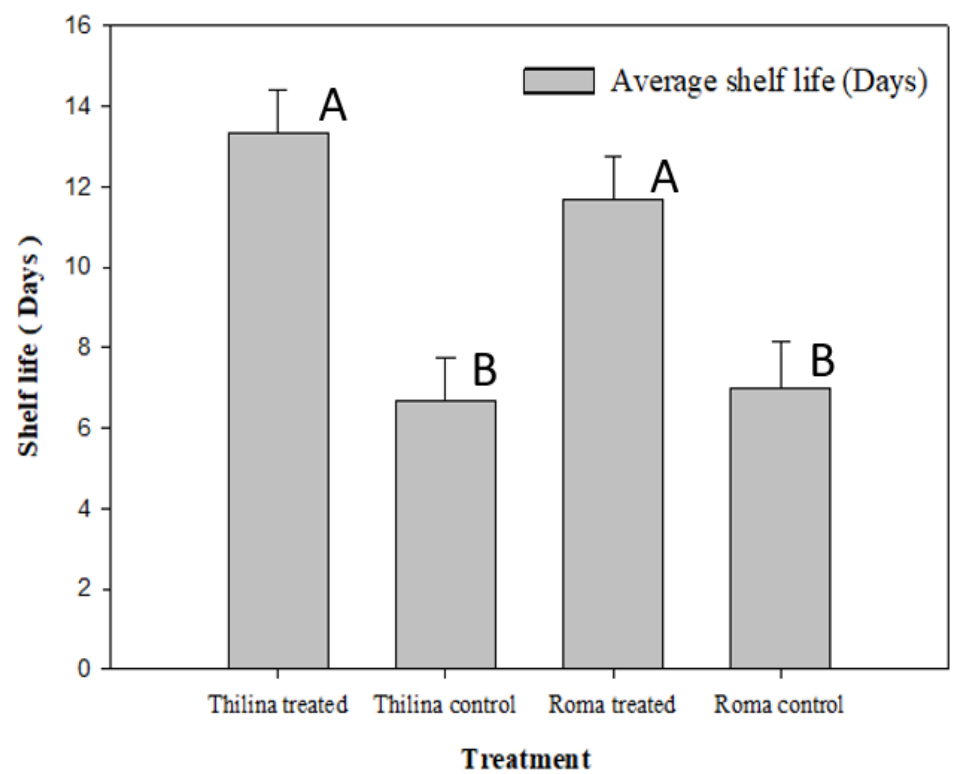

Figure 08: Effect of UV-C treatments on shelf life of tomato varieties tested, 'Thilina' and 'Roma'.

Usually fresh tomatoes have a very poor shelf life and this is a major challenge for the growers. This often forces farmers to sell their produce at a reduced price. In this study, it has shown that, UV-C treatments are effective in extending the shelf life of tomato. It is hypothesized that increased polyamine accumulation by UV-C treatment, contributes to the longer shelf life of tomatoes (Liu et al., 1993). During fruit ripening, ethylene plays a key role. However, polyamines have also been implicated in the process of controlling ripening. Further, the research findings of Liu et al., (2009) lead to the future aspects of utilizing UV-C treatments to improve tomato nutritional qualities without significantly changing the physical properties of tomatoes during storage. Research carried out by Pinheiro et al., (2015) used UV-C dosages of $0.32,0.97,2.56,4.16$ and $4.83 \mathrm{~kJ} / \mathrm{m}^{2}$ at $254 \mathrm{~nm}$, to investigate the effect on physical-chemical properties of whole tomato at $10{ }^{\circ} \mathrm{C}$ storage temperature. Although final outcome was similar to our study (i.e., UV-C treatments having an effect on physiochemical parameters but at room temperature), this result was obtained at a $10{ }^{\circ} \mathrm{C}$ storage temperature.

\section{CONCLUSION}

'Thilina' and 'Roma' varieties are more susceptible towards diseases among the tested varieties and that may be due to less natural disease resistance. All the tested tomato samples indicated the presence of antifungal compounds in their washings. Thus, fungicidal residues were remaining on the peel surface of purchased tomatoes. At $25{ }^{\circ} \mathrm{C}$, the most effective UV-C dosage against the growth of $C$. coccodes is $4.0 \mathrm{~kJ} / \mathrm{m}^{2}$. In variety 'Roma', the percentage weight loss is significantly $(\mathrm{P}<0.05)$ less when treated with UV-C. However in 'Thilina', there is no significant difference in percentage weight loss after treatments $(\mathrm{P}>0.05)$. The antioxidant activity and the total phenolic content of both treated varieties have increased against the nontreated control. The firmness has significantly retained in treated 'Thilina' variety $(\mathrm{P}<0.05)$ however; in 'Roma', there is no significant difference in firmness retention $(\mathrm{P}>0.05)$. The shelf life has significantly extended in both treated varieties $(\mathrm{P}<0.05)$. Therefore, it can be concluded that, in order to suppress anthracnose disease, for 'Thilina' variety, $4.0 \mathrm{~kJ} / \mathrm{m}^{2}$ is the most suitable dosage, while for 'Roma' variety both 3.0 and $4.0 \mathrm{~kJ} / \mathrm{m}^{2}$ dosages are preferable at $25{ }^{\circ} \mathrm{C}$ at a distance of $15 \mathrm{~cm}$. 


\section{REFERENCES}

Agarwal, S. and Rao, A.V. (2000). Tomato lycopene and its role in human health and chronic diseases. Canadian Medical Association Journal. 163(6): 739-744.

Beecher, G. (1998). Nutrient content of tomatoes and tomato products. Experimental Biological Medicine. 218: 98-100. DOI: 10.3181/00379727-218-44282a

Bu, J., Yu, Y., Aisikaer, G., \& Ying, T. (2013). Postharvest UV-C irradiation inhibits the production of ethylene and the activity of cell wall-degrading enzymes during softening of tomato (Lycopersicon esculentum L.) fruit. Postharvest Biology and Technology, 86, 337-345.

Cantwell, M. (2000). Optimum procedures for ripening tomatoes. Management of fruit ripening. Postharvest Horticultural Series. 9: 80-88.

Darras, A.I., Joyce, D.C. and Terry, L.A. (2010). Postharvest UV-C irradiation on cut Freesia hybrida L. inflorescences suppresses petal specking caused by Botrytis cinerea. Postharvest Biology and Technology. 55(3): 186-188. https://doi.org/10.1016/j.postharvbio.2009.09.005

Kanatiwela, H.M.C.K. and Adikaram, N.K.B. (2009). A TLC-bioassay based method for detection of fungicide residues on harvested fresh produce. Journal of the National Science Foundation of Sri Lanka. 37(4): 257-262. doi :10.4038/jnsfsr.v37i4.1472

Kuc', J. (2000). Development and future direction of induced systemic acquired resistance in plants. Crop Protection. 19: 859-861. DOI: 10.1016/S0261-2194(00)00122-8

Liu, C.H., Cai, L.Y., Lu, X.Y., Han, X.X. and Ying, T.J. (2012). Effect of postharvest UV-C irradiation on phenolic compound content and antioxidant activity of tomato fruit during storage. Journal of Integrative Agriculture. 11(1): 159-165. https://doi.org/10.1016/S1671-2927(12)60794-9

Liu, J., Stevens, C., Khan, V.A., Lu, J.Y., Wilson, C.L., Adeyeye, O. and Droby, S. (1993). Application of ultraviolet-C light on storage rots and ripening of tomatoes. Journal of Food Protection. 56(10): 868-873. https://doi.org/10.4315/0362-028X-56.10.868

Liu, L.H., Zabaras, D., Bennett, L.E., Aguas, P. and Woonton, B.W. (2009). Effects of UV-C red light and sun light on the carotenoid content and physical qualities of tomatoes during postharvest storage. Food Chemistry. 115: 495-500. DOI: 10.1016/j.foodchem.2008.12.042

Mditshwa, A., Magwaza, L.S., Tesfay, S.Z. and Mbili, N.C. (2017). Effect of ultraviolet irradiation on postharvest quality and composition of tomatoes: a review. Journal of Food Science and Technology. 54(10): 3025-3035. DOI: 10.1007/s13197-017-2802-6

Onik, J.C., Xie, Y., Duan, Y., Hu, X., Wang, Z. and Lin, Q. (2019). UV-C treatment promotes quality of early ripening apple fruit by regulating malate metabolizing genes during postharvest storage. PLoS ONE. 14(4): e0215472. https://doi.org/10.1371/journal.pone.0215472

Pinheiro, J., Alegria, C., Abreu, M., Gonçalves, E.M. and Silva, C.L.M. (2015). Use of UV-C postharvest treatment for extending fresh whole tomato (Solanum lycopersicum, cv. Zinac) shelf-life. Journal of Food Science and Technology. 52(8): 5066-5074. DOI: 10.1007/s13197014-1550-0

Shama, G. and Alderson, P. (2005). UV hormesis in fruits: a concept ripe for commercialization. Trends in Food Science and Technology. 16(4): 128-136. https://doi.org/10.1016/j.tifs.2004.10.001 
Tauxe, R.V. (2001). Food safety and irradiation: protecting the public from foodborne infections. Emerging Infectious Diseases. 7(3 Suppl): 516. doi: 10.3201/eid0707.017706

Terry, L.A. and Joyce, D.C. (2004). Elicitors of induced disease resistance in postharvest horticultural crops: a brief review. Postharvest Biology and Technology. 32: 1-13. https://doi.org/10.1016/j. postharvbio.2003.09.016

Wills, R., McGlasson, B., Graham, D. and Joyce, D. (2007). Postharvest: An Introduction to the Physiology \& Handling of Fruit, Vegetables and Ornamentals, CABI, Publishing, UK. 